\section{Digitalisierung in der Siedlungswasserwirtschaft}

Digitalisierung ist ein Schlagwort, welches derzeit in der Gesellschaft stark strapaziert wird. Ausgehend von Entwicklungen in der Informations- und Kommunikationstechnik (IKT) wird eine Vernetzung von Anlagen und Infrastrukturen durch den Ausbau des Breitbandinternets und Erforschung neuer Übertragungstechniken angestrebt. In der Siedlungswasserwirtschaft ist diese Technologie zwar zum Teil bereits seit Jahrzehnten im Einsatz und wird in vielfältiger Weise genutzt, jedoch haben sich die Rahmenbedingungen und die technischen Möglichkeiten in den letzten Jahren signifikant weiterentwickelt. Durch die fortschreitende Digitalisierung im Bereich Siedlungswasserwirtschaft ergeben sich auch neue Möglichkeiten für die Überwachung und Steuerung von dezentralen Anlagenteilen in Echtzeit. Durch die vermehrte Einbindung von kostengünstigen Informations- und Kommunikationstechnologien ist der aktuelle Zustand des Systems (de facto) in Echtzeit bekannt. Somit können sowohl räumlich als auch zeitlich hochaufgelöste Daten für die Umsetzung von Managementmaßnahmen in Wasserversorgung, Siedlungsentwässerung und Abwasserreinigung verwendet werden. Mit den vermehrten Einsatzmöglichkeiten wächst aber auch der mögliche Missbrauch von Informationen. Die vielfältigen Zugriffsmöglichkeiten ins System stellen ein neues Gefahrenpotenzial für die Siedlungswasserwirtschaft dar.

Die vorliegende Ausgabe der Österreichischen Wasser- und Abfallwirtschaft widmet sich mit fünf ausgewählten Fachbeiträgen dem Thema Digitalisierung in der Siedlungswasserwirtschaft und beleuchtet die Thematik von unterschiedlichen Gesichtspunkten.

Back et al. zeigen die steigende Verfügbarkeit von digitalen Daten und geben einen Überblick über georeferenzierte Daten, deren Maßstabsebenen, Verfügbarkeit und schlussendlich deren Informationsgehalt. Darüber hinaus werden Potenziale und Anwendungsmöglichkeiten für siedlungswasserwirtschaftliche Anwendungen - speziell für die Planung von grüner und blauer Infrastruktur unter Niederschlagsbelastung - untersucht.

Der Einsatz von IKT ermöglicht völlig neuartige Überwachungs- und Bewirtschaftungskonzepte. Oberascher et al. zeigen die Potenziale von innovativen, dezentralen und multifunktionalen Bewirtschaftungskonzepten durch Einbindung von Informations- und Kommunikationstechnologien, um spartenübergreifend Vorteile für Entwässerung und Trinkwasserversorgung zu erzielen. Speziell wurde eine - datentechnisch bidirektional eingebundene - smarte Regentonne entwickelt, welche seit Frühjahr 2019 am Campus Technik der Universität Innsbruck für Demonstrations- und Testzwecke betrieben wird. Die positiven Auswirkungen eines großflächigen Einsatzes wurden mittels detaillierter Simulation nachgewiesen.

Die fortschreitende Datenverfügbarkeit und steigenden Datenmengen ermöglichen zwar neue Wege der Bewirtschaftung von siedlungswasserwirtschaftlichen Systemen, es bedarf dazu aber auch neuer Ansätze zum Umgang und Einsatz dieser Daten. Die Verarbeitung von Big Data und der Einsatz von künstlicher Intelligenz stellen die Ausgangspunkte datengetriebener Modelle dar. Sappl et al. vermitteln dazu die theoretischen Grundlagen und verschaffen einen Überblick über künstliche Intelligenz und maschinelles Lernen im Bereich Siedlungswasserwirtschaft.

Neue Anwendungs- und Einsatzmöglichkeiten gehen aber auch einher mit neuen (rechtlichen) Anforderungen und technischen Risiken. Weber beleuchtet in diesem Zusammenhang die rechtlichen Aspekte der Digitalisierung in der Siedlungswasserwirtschaft. Er stellt die Verpflichtungen des Wasserversorgungsunternehmens den umfangreichen Rechten des Kunden im Zusammenhang mit der Verarbeitung von personenbezogenen Daten im Sinne der Datenschutz-Grundverordnung (DSGVO) gegenüber.

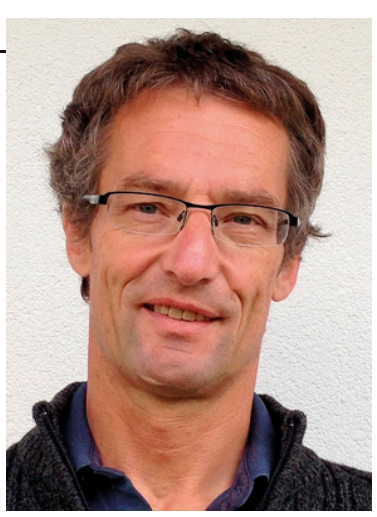

Univ.-Prof. DI Dr. W. Rauch

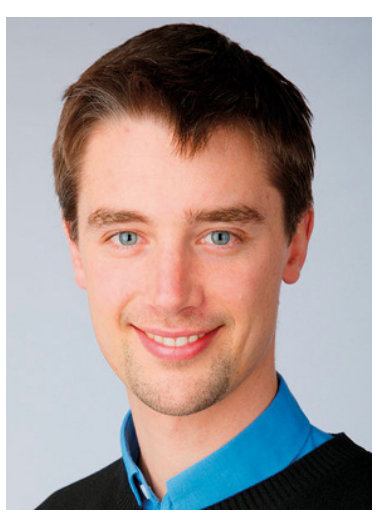

Univ.-Prof. DI Dr. R. Sitzenfrei 


\section{Editorial}

Die Sicherstellung der Versorgungs- und Betriebssicherheit siedlungswasserwirtschaftlicher Betriebe hat eine immens hohe Bedeutung. Durch den zunehmenden Einsatz der IKT steigt die Gefahr gezielter Cyberattacken auf Anlagen oder nicht zielgerichteter, systematischer Angriffe, die in Störungen des Betriebs resultieren. In diesem Zusammenhang erläutern Pointl et al. die Aspekte der IKT-Sicherheit und Cyberattacken in der österreichischen Siedlungswasserwirtschaft. Dabei wird eine systematische Schwachstellenanalyse verwendet, um potenzielle Gefährdungen auf die Versorgungssicherheit bzw. die Betriebssicherheit von Anlagen zu analysieren. Die Ergebnisse zeigen sowohl einen hohen Standard der IKT-Sicherheit der österreichischen Siedlungswasserwirtschaft, aber auch eine Reihe von Schwachstellen.

Univ.-Prof. DI Dr. W. Rauch (ه) • Univ.-Prof. DI Dr. R. Sitzenfrei

Arbeitsbereich Umwelttechnik, Institut für Infrastruktur, Universität Innsbruck, Technikerstraße 13,

6020 Innsbruck, Österreich wolfgang.rauch@uibk.ac.at

Univ.-Prof. DI Dr. R. Sitzenfrei

robert.sitzenfrei@uibk.ac.at 